\title{
Theoretical Analysis and Model Experiments on the Formation Mechanism of Channel-type Segregation*
}

\section{By Shigeo ASAI** and Iwao MUCHI**}

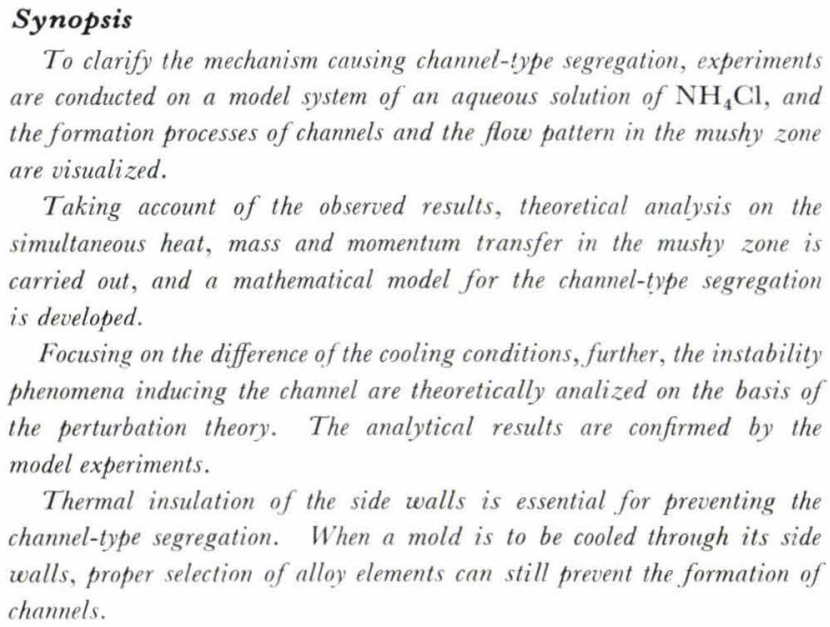

To clarify the mechanism causing channel-type segregation, experiments are conducted on a model system of an aqueous solution of $\mathrm{NH}_{4} \mathrm{Cl}$, and the formation processes of channels and the flow pattern in the mushy zone are visualized.

Taking account of the observed results, theoretical analysis on the simultaneous heat, mass and momentum transfer in the mushy zone is carried out, and a mathematical model for the channel-tvpe segregation is developed.

Focusing on the difference of the cooling conditions, further, the instability phenomena inducing the channel are theoretically analized on the basis of the perturbation theory. The analytical results are confirmed by the model experiments.

Thermal insulation of the side walls is essential for preventing the channel-type segregation. When a mold is to be cooled through its side walls, proper selection of alloy elements can still prevent the formation of channels.

\section{Introduction}

As is well known, the channel-type macrosegregations such as "V" and "A" segregations are often detected in large killed ingots. To explain the mechanism generating the macrosegregation and also to suppress its formation, many works $^{1-11)}$ have been reported.

Mehrabian and Flemings ${ }^{6)}$ presented a quantitative analysis with regard to the mechanism of centerline macrosegregation caused by the shrinkage and the density difference. The recent paper presented by Flemings ${ }^{11)}$ gave valuable information concerning the mechanism of the channel segregation. McDonald and Hunt ${ }^{4)}$ conducted a model study to visualize the channel-type segregation.

Now, in clarifying the mechanism of the channeltype segregation, the following difficulties must be overcome. Namely, the mechanism must be able to explain the complicated phenomena which are the gross sum of ones caused by the distributions of temperature, concentration and liquid fraction and also by the natural convection due to the density difference. Furthermore, no direct observation is possible in the ingot casting process of a killed steel.

In this work, the freezing experiments to demonstrate the behavior of the channel formation in the mushy zone were conducted by using aqueous solutions of ammonium chloride. Furthermore, by considering the liquid flow in the mushy zone to play an important role for the formation of macrosegregation, a mathematical model of the channel-type segregation has been developed.

\section{Experiments}

\section{Experimental Procedures}

According to the phase diagram of Fig. 1, when a solution having a concentration of ammonium chloride higher than the eutectic concentration $(19.7 \mathrm{wt} \%)$ is solidified, crystals of ammonium chloride should grow from the liquid phase. An aqueous solution of $\mathrm{NH}_{4} \mathrm{Cl}$ of $40 \%$ was used in the model test.

Experimental apparatus is shown in Fig. 2. The solution was poured into a two-dimensional mold. Both sides of the mold were in contact with two vertical chills which were cooled by a mixture of ethyl alcohol and dry ice. The freezing process was photographed every few minutes with the aid of a light source placed behind the mold.

After the completion of the freezing, the coolant was removed from the chills, and then the solidified ingot was uniformly heated by the light source. Due to the difference in the melting temperature, the higher concentration parts which correspond to the channel segregation melted earlier than the others. Thus, the string-like segregation channels were distinguished visually appearing brighter than the surrounding.

To investigate the flow pattern of liquid in the mushy zone, a small amount of potassium permanganate was placed into the mushy zone to serve as the dye tracer. The progressive dispersion of the tracer was photographed every two or three minutes.

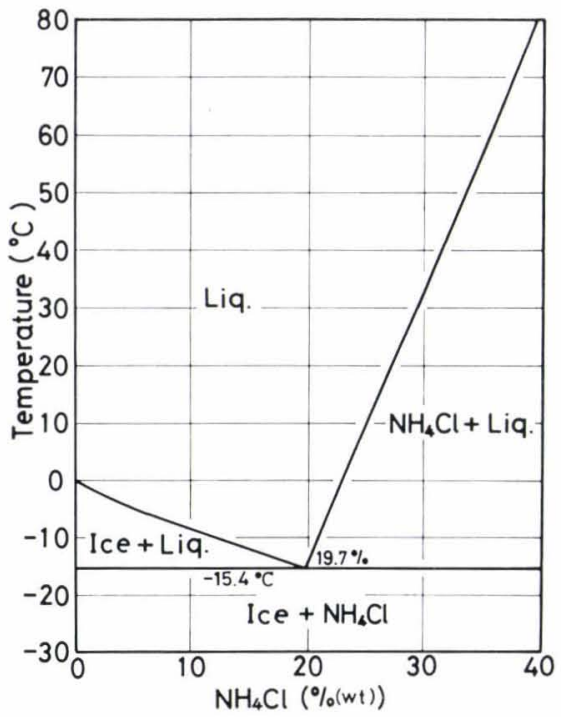

Fig. 1. Phase diagram of ammonium chloride-water system

* Originally published in Tetsu-to-Hagané, 63 (1977), 1512, in Japanese. English version received May $20,1977$.

** Department of Iron and Steel Engineering, Faculty of Engineering, Nagoya University, Furo-cho, Chikusa-ku, Nagoya 464. 


\section{Experimental Results}

1. Flow Pattern in the Mushy Zone

Photograph 1 shows the motion of fluid in the mushy zone. In the picture, the solid phase is seen as the black parts at both sides of the mold growing progressively. The mushy zone which is the white part at the central region of the mold decreases with the progress of freezing. The tracer was placed at three different positions of the mushy zone. When dissolved, the tracer dyed the liquid red, and the flow pattern was clearly delineated.

It will be noted that the red part (but appearing

(1)

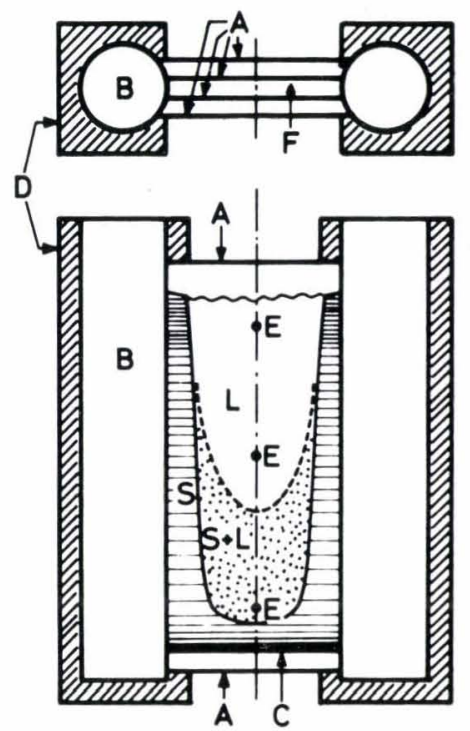

A: Transparent PVC plate.

B: Cooling box (Al pipe, Coolant: dry ice

C: Al plate,

D: Insulator.

E: Thermocouple.

$\mathrm{F}$ : $\mathrm{NH}_{4} \mathrm{Cl}$ solution to be solidified.

S: Solid phase.

L: Liquid phase,

$S+L$ : Mushy zone.

(1) plane view

(2) front view

Fig. 2. Schematic representation of experimental equipment black in the pictures) at the upper right hand side extends itself slowly both upwards and downwards, up along the surface of the solid phase and down in the central region of the mushy zone, and that the red part in the bottom, in the mean time, flows slowly first downwards then turned towards the side wall. It may be concluded then that the liquid flow in the mushy zone is to ascend along the surface of the solid phase and to descend in the central region of the mold as shown in schematic representation in Photo. 1.

\section{Formation of Channel-type Segregation}

Photograph 2 shows the transitional formation of channel-type segregation. At first, many fine lines appear at the vicinity of the surface of solid phase as shown in Photo. 2(A), and then these lines agglomerate each other and form elongated channels as seen in Photo. 2(B). A few channels survive, and the many fine lines appeared in the initial stage get disappeared as seen in Photos. 2(C) and (D). Further, the "V" type channels that never existed in Photo. $2(\mathrm{~A})$ or 2 (B) of the earlier stage become detected in the central region in Photos. 2(C) and (D). Finally, as seen in Photos. 2(E) and (F), the few survived channels are caught progressively by the growing solid phase.

Photograph 2(G) shows the channel-type segregations as melted selectively by reheating the solidified ingot. It is noted that both the shape and the position of each channel seen in Photo. $2(\mathrm{G})$ coincide with those of each channel shown in Photos. 2(B) to (D). From these observations, it has been recognized that the channels formed during freezing are resulted from macrosegregation.

\section{Consideration of the Formation Mechanism of Channel-type Segregation}

As pointed out by McDonald ${ }^{4}$ and Flemings, ${ }^{11}$ ) the flow in the mushy zone may be considered to be caused by the density difference as the driving force. Namely, if the interdendritic liquid is saturated by the solute, in other words, if the relation between the liquid temperature and the concentration can be ex-

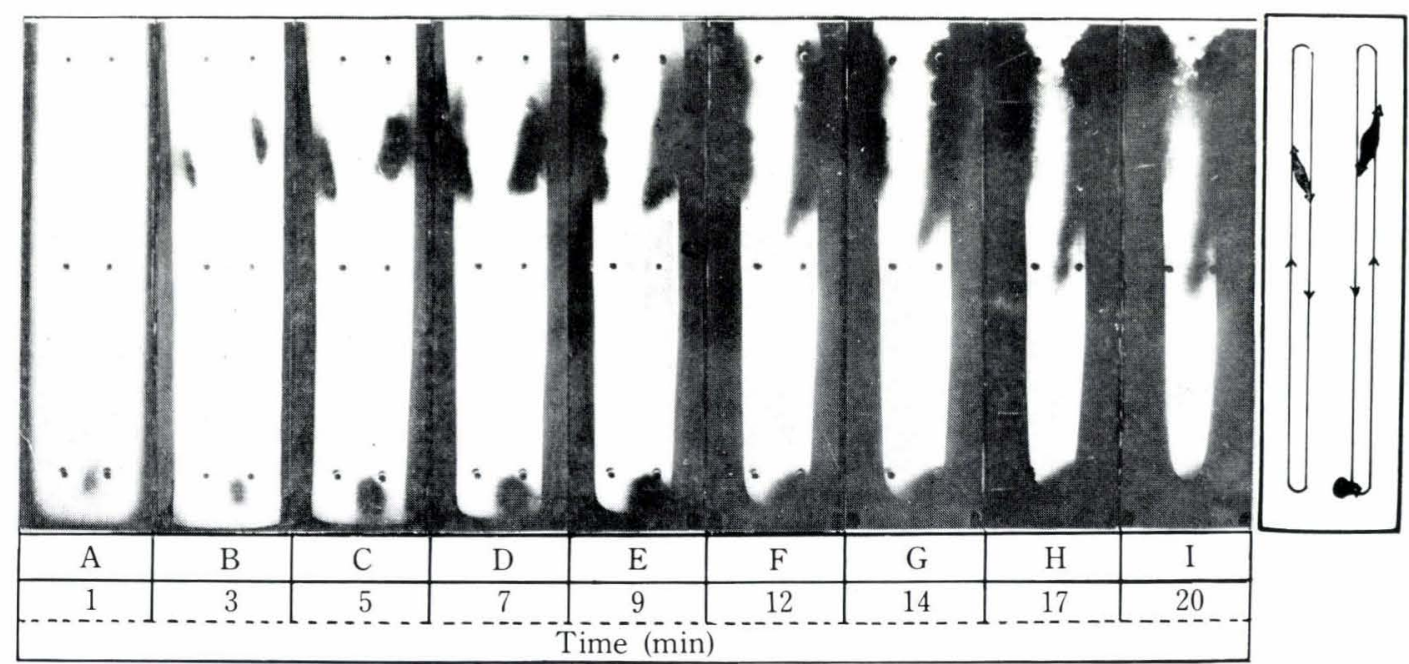

Photo. 1. Motion of fluid in the mushy zone visualized by dye tracers during freezing of an aqueous solution of $\mathrm{NH}_{4} \mathrm{Cl}$ 

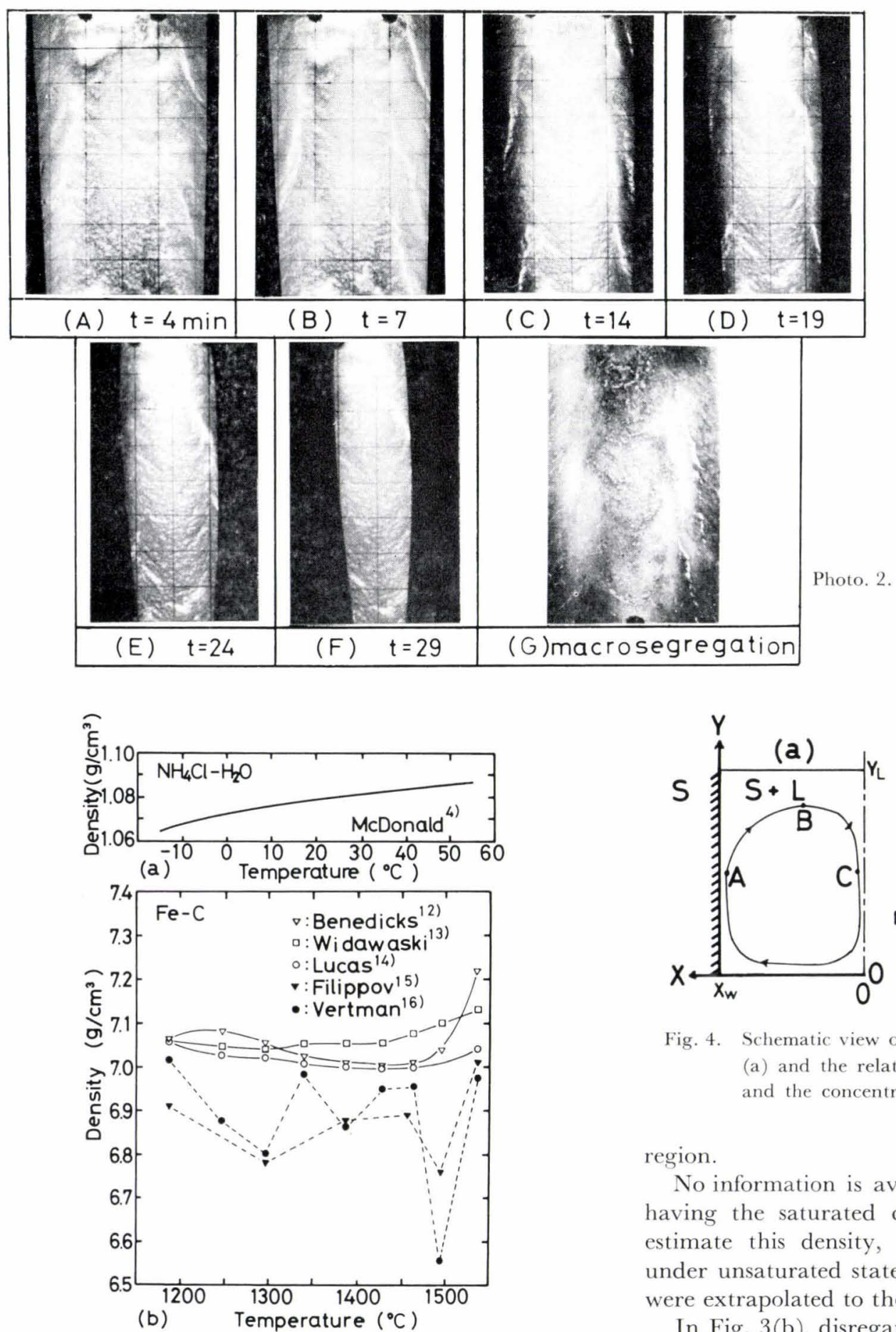

$\begin{array}{ll}\text { (a) } \mathrm{NH}_{4} \mathrm{Cl}-\mathrm{H}_{2} \mathrm{O} \text { system } & \text { (b) } \mathrm{Fe}-\mathrm{C} \text { system }\end{array}$

Fig. 3. Thermal variations in densities of saturated liquid

pressed by the liquidus line, then the density of the saturated liquid should vary in such a manner as shown in Fig. 3(a). ${ }^{4}$ This assumption may be considered as relatively reasonable.

Consequently, the density of liquid in the mushy zone decreases with the decrease in the temperature. Thus, the density at the vicinity of the surface of solid phase is lower than that at the central region of the mold owing to the temperature distribution in the mushy zone. Hence, the liquid moves upward along the surface of solid phase due to the resultant buoyancy force, and it moves downward in the central
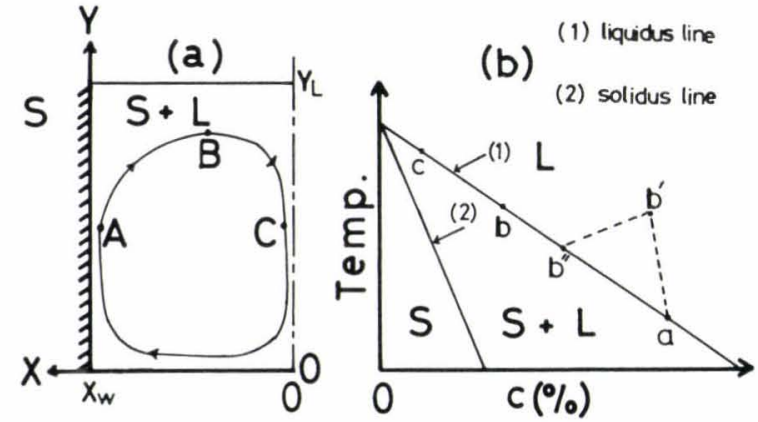

Fig. 4. Schematic view of the flow pattern in mushy zone (a) and the relationship between the temperature and the concentration of interdendritic liquid (b)

region.

No information is available for the density of steel having the saturated concentration of carbon. To estimate this density, therefore, the data obtained under unsaturated states by several investigators ${ }^{12-16}$ ) were extrapolated to the liquidus line, Fig. 3(b).

In Fig. 3(b), disregarding the black points because they are scattered too much and judging from the white points, it may be presumed that the density of steel would increase as in Fig. 3(a), and that a flow pattern similar to that of the aqueous solution of $\mathrm{NH}_{4} \mathrm{Cl}$ would appear in killed steels containing various alloy elements.

When the fluid flows as from the point $A$ to the point $B$ in Fig. 4(a), the change in the relation between temperature and concentration may be a shift not from $a$ to $b$, but from $a$ to $b^{\prime}$ as shown in Fig. 4 (b), because the rate of heat conduction is much faster than the rate of mass diffusion (i.e., Lewis number $L e=S_{c}(\operatorname{Pr} \gg 1)$ in the liquid system, particularly in the molten steel.

The state indicated by the point $b^{\prime}$ has a tendency 
to move toward the more stable state, i.e., point $b^{\prime \prime}$ on the liquidus line. This movement is accompanied by melting of solid phase in the volume element, so that the liquid fraction at the point $B$ increases. Owing to the increase in the liquid fraction, the resistance to flow at that point decreases. Consequently, the larger the flow rate at that point, the larger becomes the liquid fraction, developing an instability at that point, which, in turn, may cause the channeltype segregation.

Once a large channel has been formed, fine channels existing near the large one start disappearing progressively, since the liquid can flow more easily through the large channel than the fine ones.

The interpretation regarding the "A" type segregation as described above may be applied to the "V" type segregation in the central region of the mold. In this region, the flow moves downward from the point $B$ to the point $C$ as illustrated in Fig. $4(\mathrm{a})$, so that the " $\mathrm{V}$ " type pattern may be formed there.

The "V" type segregations that are formed in a killed steel ingot at a more or less regular interval may be closely related to the periodicity at which these fine channels existing in the neighborhood of large ones progressively disappear.

The fact that the "V" type segregation was observed even in the $\mathrm{NH}_{4} \mathrm{Cl}-\mathrm{H}_{2} \mathrm{O}$ system, in which freezing takes place, contrary to steels, accompanied by expansion of volume, suggests that the "V" type segregation is surely caused by the fluid flow due to the density difference.

In conclusion, it may be presumed from the model studies described above that the channel-type segregation occurring in the steel casting process is caused by the fluid flow moving from the interface between the solid shell and the mushy zone to the center, and that such fluid flow will be induced whenever the fluid density decreases with the progress of freezing, namely in steels containing alloy elements lighter than iron. This conclusion may be considered verified by Smeltzer, ${ }^{17)}$ who found empirically that the freckle of stainless steel was prevented by decreasing the concentration of silicon, and by Suzuki, ${ }^{10)}$ who found in a pilot scale study that the "A" type segregation was promoted by adding an element lighter than iron (e.g., vanadium) and that it was surpressed by adding a heavier one (e.g., molybdenum).

\section{Mathematical Model of Channel-type Segre- gation}

From the viewpoint that the fluid flow mentioned above affects strongly the formation of the channeltype segregation, the following theoretical analysis has been developed.

Concerning the liquid in the mushy zone, the equation of continuity is written as follows:

$$
\partial \varepsilon / \partial t+\partial(\varepsilon u) / \partial X+\hat{\partial}(\varepsilon v) / \partial Y=0
$$

By assuming the quasi-steady state, Eq. (1) is rewritten as Eq. $\left(1^{\prime}\right)$ :

$$
\partial(\varepsilon u) / \partial X+\partial(\varepsilon v) / \partial Y=0
$$

Assuming that the Darcy's law can describe the states of liquid flow in the mushy zone, the equations of motion can be derived as Eqs. (2) and (3).

$$
\begin{aligned}
& u=-(k / \mu \varepsilon)(\partial P / \partial X) \quad \ldots \ldots \ldots \ldots \\
& v=-(k / \mu \varepsilon)(\partial P / \partial Y)-(k \rho g / \mu \varepsilon)
\end{aligned}
$$

where $k$ is the permeability which depends on the liquid fraction and the size of pore in the mushy zone. Strictly speaking, the mushy zone is anisotropic. But, for the sake of simplicity, the mushy zone may well be assumed as an inhomogeneous and isotropic medium. Now, on the basis of the Kozeny-Carman equation, $k$ can be expressed as Eq. (4).

$$
k=\left(1 / 5 S_{v}^{2}\right)\left\{\varepsilon^{3} /(1-\varepsilon)^{2}\right\}
$$

where $S_{v}$ designates the volumetric specific surface area (surface area per unit volume of solid material). Assuming the geometrical shape of a dendrite arm to be a cone having a height $h$ and $a$ diameter of the base $S_{11}, S_{v}$ can be approximately expressed as Eq. (5).

$$
S_{v}=\left(6 / S_{11}\right) \sqrt{ } 1+\left(S_{11} / 2 h\right)^{2}=\left(6 / S_{11}\right)^{*}
$$

Taking the heat balance around a volume element of the solid and liquid mixture in the mushy zone,

$$
\begin{aligned}
& \partial T / \partial t+\varepsilon u(\partial T / \partial X)+\varepsilon v(\partial T / \partial Y)=\left(\kappa / C_{p} \rho\right)\left\{\left(\partial^{2} T / \partial X^{2}\right)\right. \\
& \left.\left.\quad+\left(\partial^{2} T / \partial Y^{2}\right)\right\}+((-\lrcorner H) / C_{p}\right)(\partial \varepsilon / \partial t) \ldots \ldots \ldots \ldots \ldots \ldots \ldots \ldots \ldots \ldots \ldots \ldots \ldots
\end{aligned}
$$

With regard to the liquid in the mushy zone, the mass balance equation is expressed by Eq. (7).

$$
\begin{aligned}
& \varepsilon(\partial C / \partial t)+\varepsilon u(\partial C / \partial X)+\varepsilon v(\partial C / \partial Y)=\varepsilon D\left\{\left(\partial^{2} C / \partial X^{2}\right)\right. \\
& \left.\quad+\left(\partial^{2} C / \partial Y^{2}\right)\right\}+(1-K) C(\partial(1-\varepsilon) / \partial t) \ldots \ldots \ldots \ldots \ldots \ldots
\end{aligned}
$$

The diffusion terms in Eq. (7) (i.e., the first term in the right hand side) is so small (Péclet number for mass transfer $=(R e)(S c) \gg 1)$ that the mass balance equation may be rewritten as Eq. (8).

$$
\begin{gathered}
\partial C / \partial t+u(\partial C / \partial X)+v(\partial C / \partial Y) \\
\quad=-\{(1-K) C / \varepsilon\}(\partial \varepsilon / \partial t) \ldots
\end{gathered}
$$

Assuming that the relations between the temperature and the concentration of interdendritic liquid may be expressed by those based on the liquidus line,

$$
T=T_{0}+m C
$$

Eliminating $C$ from Eqs. (8) and (9),

$$
\begin{aligned}
\partial \varepsilon / \partial t= & \left\{\varepsilon /(1-K)\left(T_{0}-T\right)\right\}\{\partial T / \partial t \\
& +(1 / \varepsilon)(\partial \psi / \partial Y)(\partial T / \partial X) \\
& -(1 / \varepsilon)(\partial \psi / \partial X)(\partial T / \partial Y)\}
\end{aligned}
$$

* This approximate expression holds for arm lengths of $h \gg\left(S_{\mathrm{II}} / 2\right)$, and even in an extreme case such as $h=S_{1 \mathrm{I}}, S_{v}$ is larger only by about $12 \%$. 


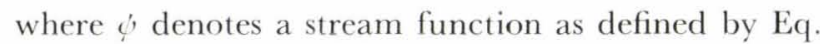
(11).

$$
u=(1 / \varepsilon)(\partial \psi / \partial Y), \quad v=-(1 / \varepsilon)(\partial \psi / \partial X)
$$

The expression for the stream function is obtained by eliminating $(\partial P / \partial X)$ and $(\partial P / \partial Y)$ from Eqs. $\left(1^{\prime}\right)$, (2) and (3).

$$
\begin{aligned}
& \partial^{2} \psi / \partial X^{2}+\partial^{2} \psi / \partial Y^{2}+\{\partial(\ln \mu / k) / \partial X\}(\partial \psi / \partial X) \\
& \quad+\{\partial(\ln \mu / k) / \partial Y\}(\partial \psi / \partial Y)=-\beta \rho g(\partial T / \partial X)(k / \mu)
\end{aligned}
$$

where $\beta$ is the volumetric expansion coefficient of the saturated liquid upon freezing.

Finally, by solving Eqs. (6), (10) and (12) simultaneously under appropriate boundary conditions, the profiles of velocity, temperature and liquid fraction in the mushy zone can be obtained for any instant during the freezing.

\section{Analysis of the Instability Causing Channel- type Segregation}

Here, the condition inducing the instability causing the channel-type segregation will be quantitatively investigated by using the mathematical model.

By eliminating $(\partial T / \partial t)$ from Eqs. (6) and (10), Eq. (13) can be derived as follows:

$$
\begin{aligned}
{\left[(1-K)\left(T_{0}-T\right) / \varepsilon+\Delta H / C_{p}\right](\partial \varepsilon / \partial t) } \\
=\left(\kappa / C_{p} \rho\right)\left\{\left(\partial^{2} T / \partial X^{2}\right)+\left(\partial^{2} T / \partial Y^{2}\right)\right\} \\
\quad+(1-\varepsilon) u(\partial T / \partial X)+(1-\varepsilon) v(\partial T / \partial Y)
\end{aligned}
$$

Since the terms in brackets of the left hand side of Eq. (13) are always positive, the sign of $(\partial \varepsilon / \partial t)$ can be readily determined by the sign of the right hand side.

\section{The Case where Both the Top and the Bottom of Mold are Insulated}

In this case, Eq. (13) can be reduced to Eq. (14), since the temperature gradient in the $X$-direction is much larger than that in the $Y$-direction.

$$
\begin{aligned}
& {\left[(1-K)\left(T_{0}-T\right) / \varepsilon+\Delta H / C_{p}\right](\partial \varepsilon / \partial t)} \\
& \quad=\left(\kappa / C_{p} \rho\right)\left(\partial^{2} T / \partial X^{2}\right)+(1-\varepsilon) u(\partial T / \partial X)
\end{aligned}
$$

If the direction of $X$-axis is taken as shown in Fig. 4(a), the signs of $\left(\partial^{2} T / \partial X^{2}\right)$ and $(\partial T / \partial X)$ are kept negative during the freezing. In the case of $u>0$, that is, when the liquid flows in the direction opposite to the arrow shown in Fig. 4(a), the sign of $(\partial \varepsilon / \partial t)$ is always negative during the freezing. Namely, in this case, the liquid fraction decreases, or the freezing proceeds steadily, and the system remains stable.

On the other hand, in the case of $u<0$, that is, when the liquid flows in the arrow direction $(A \rightarrow$ $\rightarrow B \rightarrow C$ ) as shown in Fig. 4(a), there emerges a possibility that the sign of $(\partial \varepsilon / \partial t)$ becomes positive.

Namely, in the case of $u \geq 0:(\partial \varepsilon / \partial t)<0$, thus the system will be stable. In the case of $u<0$ : if $(1-\varepsilon)$ $u(\partial T / \partial X)|>|\left(\kappa / C_{p} \rho\right)\left(\partial^{2} T / \partial X^{2}\right) \mid$, then $(\partial \varepsilon / \partial t)>0$, thus the system will be unstable, and if $(1-\varepsilon) u(\partial T / \partial X)$ $<\left(\kappa / C_{p} \rho\right)\left(\partial^{2} T / \partial X^{2}\right)$, then $(\partial \varepsilon / \partial t)<0$, thus it will be quasi-stable.

\section{The Case where the Side Walls of Mold are Insulated}

In this case, Eq. (13) can be reduced to Eq. (15), since the temperature gradient in the $Y$-direction is much larger than that in the $X$-direction.

$$
\begin{aligned}
& {\left[(1-K)\left(T_{0}-T\right) / \varepsilon+\Delta H / C_{p}\right] \cdot(\partial \varepsilon / \partial t)} \\
& \quad=\left(\kappa / C_{p} \rho\right)\left(\partial^{2} T / \partial Y^{2}\right)+(1-\varepsilon) v(\partial T / \partial Y)
\end{aligned}
$$

If the condition of $(\partial T / \partial X)=0$ could be strictly kept during the freezing, no natural convection can take place since the solution of $u=v=0$ is readily obtained from Eqs. (11) and (12). Then, Eq. (15) is reduced to $\mathrm{Eq} .(16)$.

$$
\begin{aligned}
& {\left[(1-\kappa)\left(T_{0}-T\right) / \varepsilon+\Delta H / C_{p}\right] \cdot(\partial \varepsilon / \partial t)} \\
& \quad=\left(\kappa / C_{p} \rho\right)\left(\partial^{2} T / \partial Y^{2}\right) \ldots \ldots \ldots \ldots \ldots \ldots \ldots
\end{aligned}
$$

The sign of $\left(\partial^{2} T / \partial Y^{2}\right)$ is kept negative due to the convex profile of the temperature so that the sign of $(\partial \varepsilon / \partial t)$ is always negative in Eq. (16). Thus, the system can be maintained stable. However, this is valid only under the condition of $(\partial T / \partial X)=0$, which is unrealistic. This condition is so easily disturbed that it is hardly maintained through freezing in practice.

Thus, it is necessary to examine the nature of the disturbance, because, if magnified, it can induce the formation of the channel-type segregation. In order to examine whether the disturbance in temperature increases or decreases with the progress of solidification, the perturbation theory is applied as followings.

As the condition of $|\partial T / \partial Y| \gg|\partial T / \partial X|$ can be kept by means of adiabatic side walls, Eq. (15) may be considered approximately valid even if the disturbance exsists.

Supposing that $(\partial \varepsilon / \partial X)$ and $(\partial \varepsilon / \partial Y)$ are small enough to be neglected, Eq. (17) is obtained by eliminating $(\partial P / \partial X)$ and $(\partial P / \partial Y)$ from Eqs. (1'), (2) and (3).

$$
\partial v / \partial X-\partial u / \partial Y=(k g \rho \beta / \mu \varepsilon) \cdot(\partial T / \partial X) .
$$

When a disturbance is introduced, the temperature is expressed as Eq. (18).

$$
T=T_{o}+T^{\prime}
$$

where $T_{0}$ denotes the temperature before perturbed, and $T^{\prime}$ designates a small disturbance.

If there is no disturbance, $v=u=0$ from Eqs. (11) and (12) because $T=T_{o}$ and $\left(\partial T_{o} / \partial X\right)=0$. Thus, Eq. (19) is obtained from Eq. (6).

$$
\partial T_{o} / \partial t=\left(\kappa / C_{p} \rho\right) \cdot\left(\partial^{2} T_{o} / \partial Y^{2}\right)+(-\Delta H) / C_{p} \cdot(\partial \varepsilon / \partial t) \ldots
$$

If, due to the disturbance, $u$ has become from zero to $u^{\prime}$, and $v$ from zero to $v^{\prime}, \mathrm{Eq}$. (20) is obtained by substituting $T$ given by Eq. (18) and $v^{\prime}$ into Eq. (6).

$$
\begin{aligned}
\partial T_{o} / \partial t+\partial T^{\prime} / \partial t+\varepsilon v^{\prime}\left(\partial T_{o} / \partial Y+\partial T^{\prime} / \partial Y\right) \\
=\left(\kappa / C_{p} \rho\right) \cdot\left(\partial^{2} T_{o} / \partial Y^{2}+\partial^{2} T^{\prime} / \partial Y^{2}\right) \\
\quad+(-\Delta H) / C_{p} \cdot(\partial \varepsilon / \partial t) \quad \ldots \ldots \ldots \ldots \ldots \ldots \ldots \ldots \ldots \ldots \ldots \ldots
\end{aligned}
$$



(21).

In the same manner, Eq. (17) is rewritten as Eq.

$$
\begin{aligned}
\partial v^{\prime} \mid \partial X-\partial \hat{u} u^{\prime} / \partial Y & =(k g \rho \beta / \mu \varepsilon) \cdot\left(\partial T_{o} / \partial X+\partial T^{\prime} / \partial X\right) \\
& =(k g \rho \beta / \mu \varepsilon) \cdot\left(\partial T^{\prime} / \partial X\right) \ldots \ldots \ldots \ldots \ldots
\end{aligned}
$$

In view of the fact that the channel-type segregations are string-like and develop nearly vertically rather than horizontally, the variation of $v^{\prime}$ in the horizontal direction may be considered to be much larger than that of $u^{\prime}$ in the vertical direction. Namely, as the relation shown by Eq. (22) is held, Eq. (21) can be reduced to Eq. (23)

$$
\begin{array}{r}
\left|\partial v^{\prime}\right| \partial X|\gg| \partial u^{\prime}|\partial Y| \ldots \ldots . \\
\partial v^{\prime} / \partial X=(k g \rho \beta / \mu \varepsilon) \cdot\left(\partial T^{\prime} / \partial X\right)
\end{array}
$$

Considering that the coefficient of Eq. (23), $(\mathrm{kg} \rho \mathrm{\beta} /$ $\mu \varepsilon)$, is weakly affected by the disturbance $\left(T^{\prime}\right)$, Eq. (24) is obtained by integrating Eq. (23) under an initial condition of $v^{\prime}=0$ at $T^{\prime}=0$, which is the state before the introduction of the disturbance.

$$
v^{\prime}=(k g \rho \beta / \mu \varepsilon) T^{\prime}
$$

By substituting Eq. (24) into Eq. (20), and subtracting Eq. (19), then neglecting the small term $T^{\prime}\left(\partial T^{\prime} \mid \partial Y\right)$ on the basis of the relation of $T_{0} \gg T^{\prime}$, Eq. (25) is finally derived.

$$
\begin{array}{ll} 
& \partial^{2} T^{\prime} / \partial Y^{2}-\xi T^{\prime}-(1 / \lambda)\left(\partial T^{\prime} / \partial t\right)=0 \quad . . \\
\text { where, } \quad & \xi=-\left(k g C_{p} \rho / \mu \kappa\right) \cdot(\partial \rho / \partial T) \cdot\left(\partial T_{o} / \partial Y\right) \\
& \lambda=\kappa / C_{p} \rho .
\end{array}
$$

By solving Eq. (25) under the following conditions, Eq. (26) is obtained.

Initial condition: $T^{\prime}=T_{i}^{\prime} \cdot \delta(Y)$ at $t=0$

Boundary condition: $\quad T^{\prime}=0$ at $Y= \pm \infty$ at $t>0$

$$
\begin{aligned}
T^{\prime}= & \left(T_{i}^{\prime} / 4\right) \sqrt{\xi}\{\exp (\sqrt{\xi} Y) \operatorname{erfc}(\sqrt{ } \lambda \xi t+Y / 2 \sqrt{\lambda} t) \\
& +\exp (-\sqrt{\xi} Y) \operatorname{erfc}(\sqrt{\lambda \xi t}-Y / 2 \sqrt{\lambda t})\} \\
& +\left(T_{i}^{\prime} / 2 \sqrt{\pi \lambda t}\right) \exp \left\{-\left(Y^{2} / 4 \lambda t+\lambda \xi t\right)\right\} \ldots \ldots . .(2
\end{aligned}
$$

The initial condition implies that a initial small disturbance $T_{i}^{\prime}$ suddenly appears at $Y=0$, while the boundary condition means that no disturbance exists at $Y= \pm \infty$.

By Eq. (26), it is possible to know whether the disturbance $T^{\prime}$ increases or decreases with the progress of freezing. Namely;

(1) The case of $\xi>0$

$T^{\prime} \rightarrow 0$ is at $t \rightarrow \infty$ from Eq. (26), meaning that the system is stable and the channel-type segregation will not be formed. The condition of $\xi>0$ can be replaced by the condition of $(\partial \rho / \partial T) \cdot\left(\partial T_{o} / \partial Y\right)<0$, since the value of $\left(k g C_{p} \rho / \mu \kappa\right)$, to be determined by the physical property, is positive.

Then, the condition of $\xi>0$ corresponds to the state of $\left(\partial T_{o} / \partial Y\right)<0$, when the density of interdendritic liquid increases with the increase in the temperature $((\partial \rho / \partial T)>0)$. Also, it corresponds to the state of $\left(\partial T_{o} / \partial Y\right)>0$, when $((\partial \rho / \partial T)<0)$. Here, $\left(\partial T_{o} / \partial Y\right)<0$ implies the mold is being cooled from the top, and $\left(\partial T_{o} / \partial Y\right)>0$ suggests cooling from bottom.

As is mentioned before, the channel-type segregation may be formed when $(\partial \rho / \hat{\partial} T)>0$ in an ordinary mold which is cooled from the side walls. Thus, this segregation may be prevented by cooling from the top and also by insulating the side walls.

(2) The case of $\xi<0$

$T^{\prime} \rightarrow \infty$ is from Eq. (26), meaning that the system is unstable and there is a possibility the channel-type segregations may be formed. That is, channels may be created if the disturbance propagates quickly, but the disturbance may give rise to no channels if it grows slowly in comparison with the partial solidification velocity. The propagating velocity of the disturbance is controlled by a dimensionless number $\left(\xi \lambda t_{p}\right)$, which is found in Eq. (26). Another formulation of this number is given in Eq. (27)

$$
\begin{aligned}
\xi \lambda t_{p} & =\left\{k g \beta S_{\mathrm{II}}^{2}\left(\partial T_{o} / \partial Y\right) /(\mu / \rho)^{2}\right\} /\left(\rho V S_{\mathrm{II}} / \mu\right) \\
& =(1 / 2) G r^{\prime} \mid \operatorname{Re} \quad \ldots \ldots \ldots \ldots \ldots \ldots \ldots \ldots \ldots \ldots \ldots \ldots \ldots \ldots \ldots \ldots \ldots \ldots \ldots
\end{aligned}
$$

where $V=S_{I I} / 2 t_{p}$ is the partial solidification velocity, and $t_{p}$ is the partial solidification time.

\section{Experimental Demonstrations}

To examine the analytical results regarding the formation of the channel-type segregation, model experiments were conducted by using an aqueous solution of $\mathrm{NH}_{4} \mathrm{Cl}(40 \mathrm{wt} \%)$. As shown in Fig. 3(a), the density of this solution varies as $(\hat{\partial} \rho / \hat{\partial} T)>0$ under the saturated condition.

According to the analytical results, the channeltype segregation will be produced by cooling from the bottom or from the side walls of the mold, whereas it will be prevented by cooling from the top. On the basis of these results, the following three different cooling conditions were employed.

I) Cooling from the top of mold having the adiabatic side walls

II) Cooling from the bottom of mold having the adiabatic side walls

III) Cooling from the side walls of mold insulating the top and the bottom

The results of the experiment (I) is shown in Photo. 3. Photographs 3(A) to (E) and (F) were taken during the freezing and after the completion of solidification, respectively, in the same way described in the experimental procedure. The upper black part corresponds to the solid phase, and the mushy zone looks gray at the bottom. In the all pictures no channel-type segregation can be detected. Instead of the channel-type, a horizontal segregation line like a band type segregation can be seen in Photo. 3(F). The white part seen along the left side wall is not a segregation line, but is one that was resulted from the non-uniform heating after the completion of solidification.

Photograph 4 shows the results of the experiment (II). The observed structures were similar to those of the experiment (I), except one channel-line is seen 

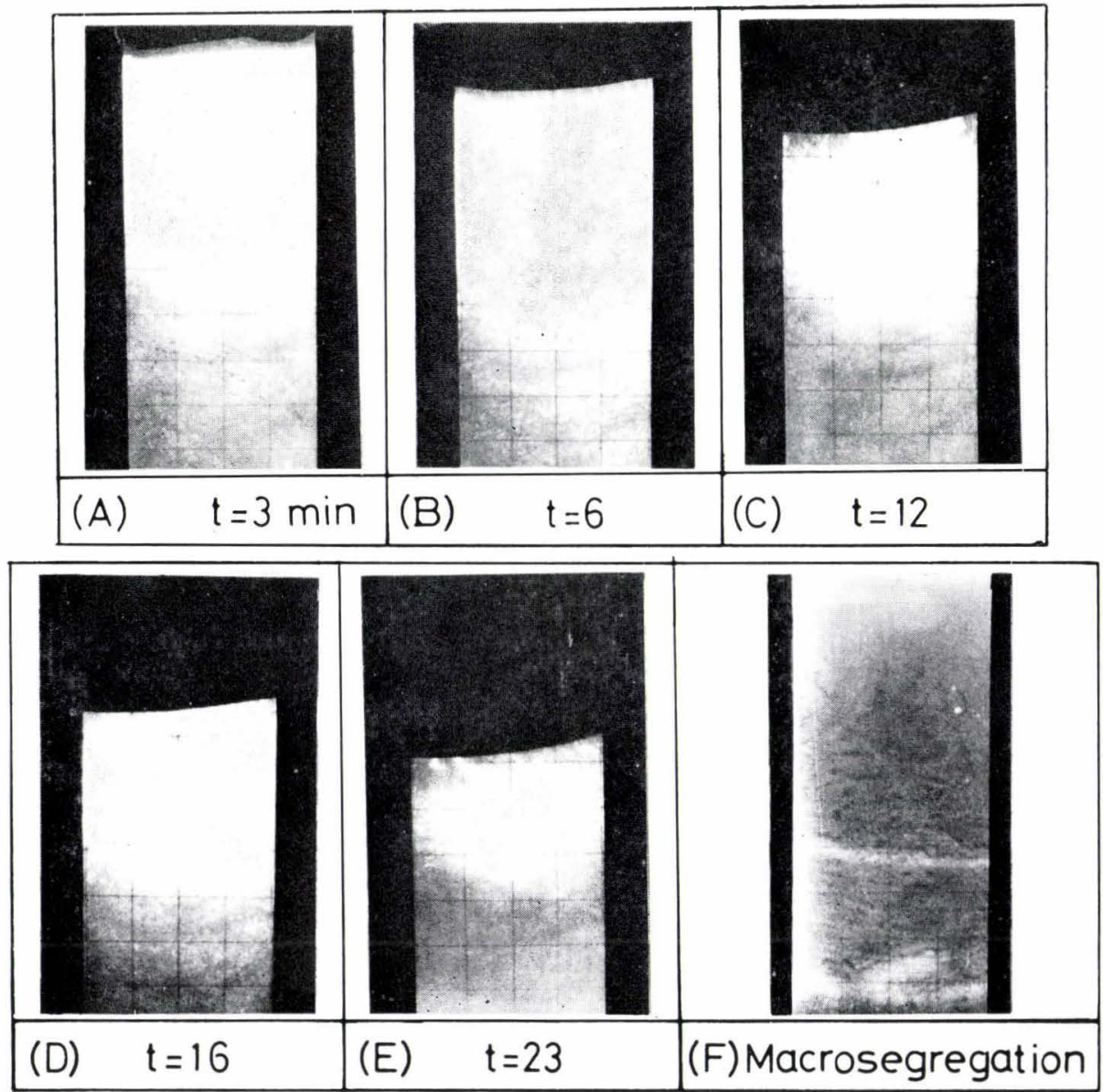

Coolant: liquid nitrogen Photo. 3. Transitional freezing in the mushy zone when cooled from the top of a mold
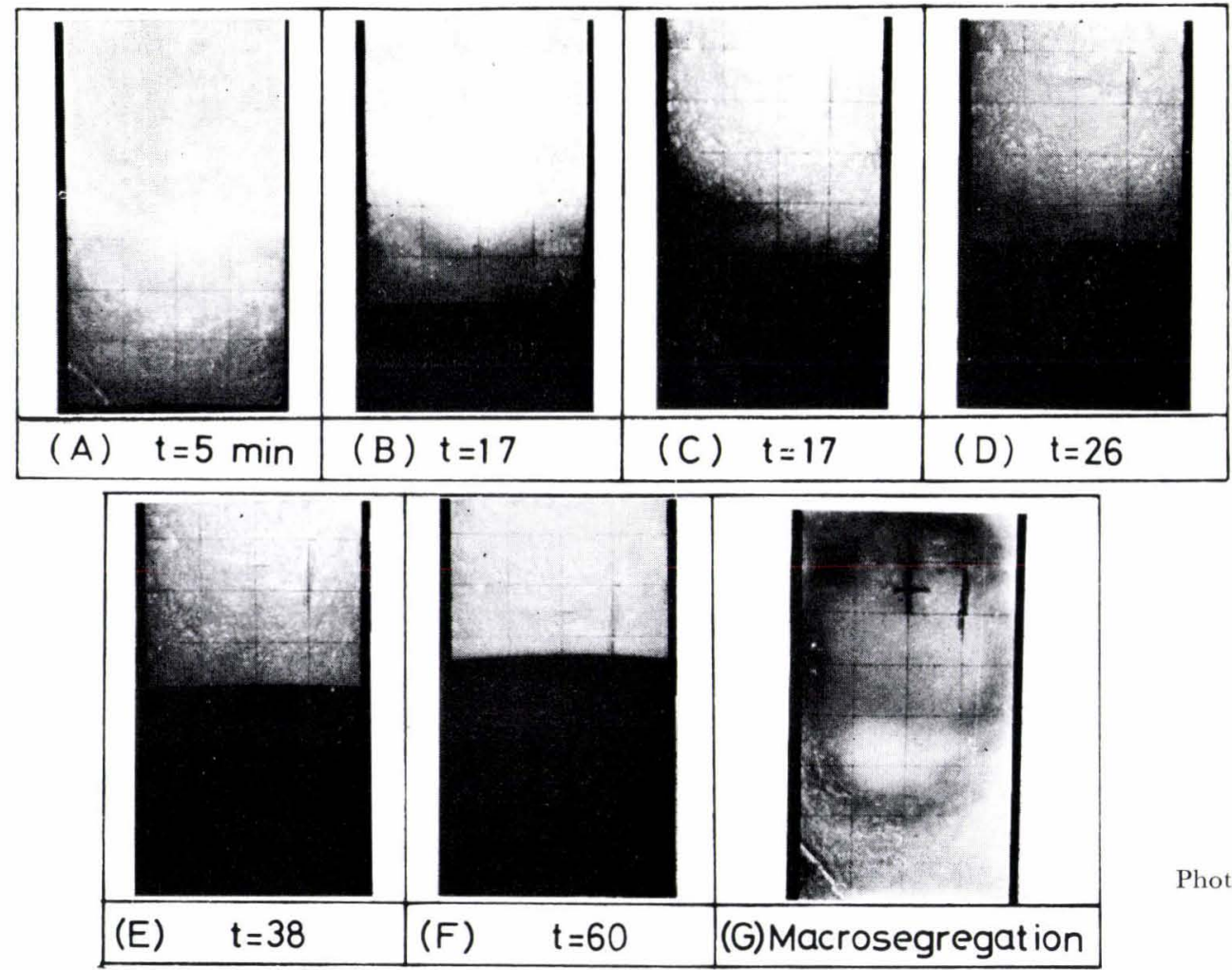

Coolant: liquid nitrogen

Photo. 4. Transitional freezing in the mushy zone when cooled from the bottom of a mold

in Photo. 4(A) and (G). In this case, it may be considered that the propagating velocity of the disturbance was not fast enough to produce many channels

The results of the experiment (III) were already shown in the Photo. 2. The extensive channel formation was the features.

It is noted that the set of the experimental observations agree quite well with the analytical results. 


\section{Procedure for Computation and the Results}

A mathematical model presented in this paper consists of the non-linear partial differencial equations of the parabolic type (Eqs. (6) and (10)) and the elliptic type (Eq. (12)). The numerical technique used to solve Eqs. (6) and (10) was the Implicit method, whereas the Gauss-Seidel method was applied to Eq. (12). Owing to this complication of having to use different type equations, a typical run for a real time of $16 \mathrm{sec}$ demands a substantial computing time of about $125 \mathrm{sec}$ (C.P.U.) on the FACOM 230-60 (the degital computer of the Nagoya University).

The result computed with regard to the variations of the liquid fraction is illustrated in Fig. 5. Random numbers having the mean value of 0.5 and a standard deviation $(\sigma)$ of 0.08 were given as the initial values of liquid fraction in the mushy zone on each mesh point $(21 \times 11$ points $)$ employed for computation. Other data and the boundary conditions applied to these computations are listed in Table 1. In Fig. 5, the initial values of liquid fraction are expressed by the short lines " - " and the values computed for a $25 \mathrm{sec}$ of elapsed time are expressed by the long lines "— ".

Figure 5 shows that, with the progress of freezing, the values of $\varepsilon$ at the most of the given mesh points decrease. This means that the freezing proceeds progressively. It may be noticed, however, that at a few points, the liquid fraction increases. The phenomenon means instability and the occurrence of the channel-type segregation.

Figure 6 shows the relation between $\log \left(S_{\mathrm{II}}\right)$ and the elapsed time $(t)$ at which the instability begins to appear at any point in the system. The mean value of the initial liquid fraction was given as 0.4 . It is found from Fig. 6 that the smaller the $S_{\text {II }}$ and $\sigma$, the longer the system remains stable. When $\sigma$ is less than a certain critical value 0.05 for this computation), the system will never be unstable during freezing.
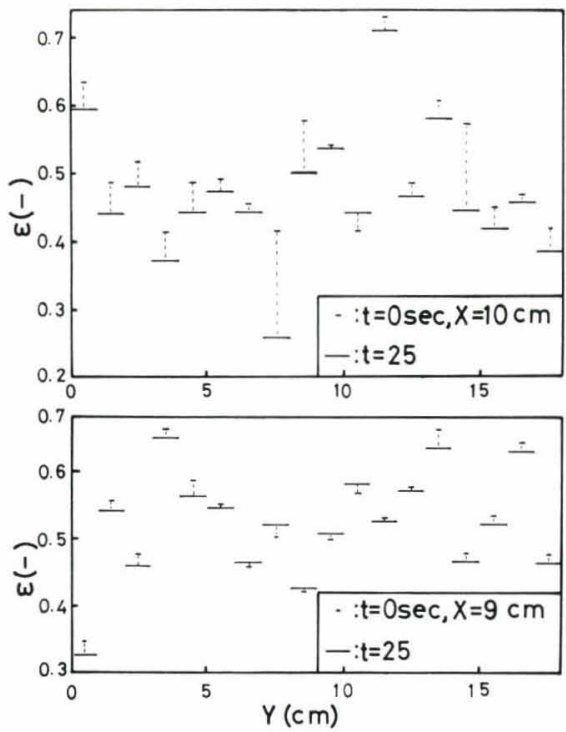

Fig. 5. Calculated results concerning the transitional variations of the local liquid fractions in mushy zone
Recently, Suzuki and Miyamoto ${ }^{9,10)}$ suggested that the differences between the microstructure of the vacuum carbon deoxidized steels and that of the ordinary vacuum casted steels deoxidized by $\mathrm{Si}-\mathrm{Mn}$ or $\mathrm{Si}-\mathrm{Al}$ may be related with the occurrence of the channel-type segregation. That is, in the latter steels, the "A" type segregation is more often detected than in the former, in which the dendrite arm spacings are distributed more uniformly than in the latter. Their suggestion is in qualitative agreement with the computed results shown in Fig. 6.

\section{Conclusion}

On the basis of model tests visualizing the flow pattern in the mushy zone and also the progressive formation of the channel-type segregation, theoretical analysis of simultaneous heat, mass and momentum transfer has been developed.

The instability of the system which might lead to the occurrence of the channel-type segregation has been qualitatively considered. Furthermore, the in-

Table 1. Boundary conditions and data for numerical computation

Boundary conditions

$\begin{array}{ll}\dot{\phi}=0, \partial T / \partial X=0 & \text { at } X=0, \quad 0<Y<Y_{L} \\ \phi=0, T=-15^{\circ} \mathrm{C} & \text { at } X=X_{w}, \quad 0<Y<Y_{L} \\ \phi=0, T=-15^{\circ} \mathrm{C} & \text { at } Y=0, \quad 0<X<X_{w} \\ \phi=0, \partial T / \partial Y=0 & \text { at } Y=Y_{L}, \quad 0<X<X_{w}\end{array}$

Physical properties of $\mathrm{NH}_{4} \mathrm{Cl}-\mathrm{H}_{2} \mathrm{O}$ system

$\begin{aligned} C_{p} & =0.75 \mathrm{cal} / \mathrm{g}^{\circ} \mathrm{C}, & & \lambda=0.016 \mathrm{~cm}^{2} / \mathrm{sec} \\ \Delta H & =71 \mathrm{cal} / \mathrm{g}, & & \mu=0.01 \mathrm{~g} / \mathrm{cm} \cdot \mathrm{sec} \\ T_{0} & =359.5^{\circ} \mathrm{C}, & & \rho \beta=-4.0 \times 10^{-4} \mathrm{~g} / \mathrm{cm}^{3} \cdot{ }^{\circ} \mathrm{C}\end{aligned}$

Data given

$K=0.3, \quad S_{\mathrm{II}}=0.01 \mathrm{~cm}, \quad T_{i}=20^{\circ} \mathrm{C}$

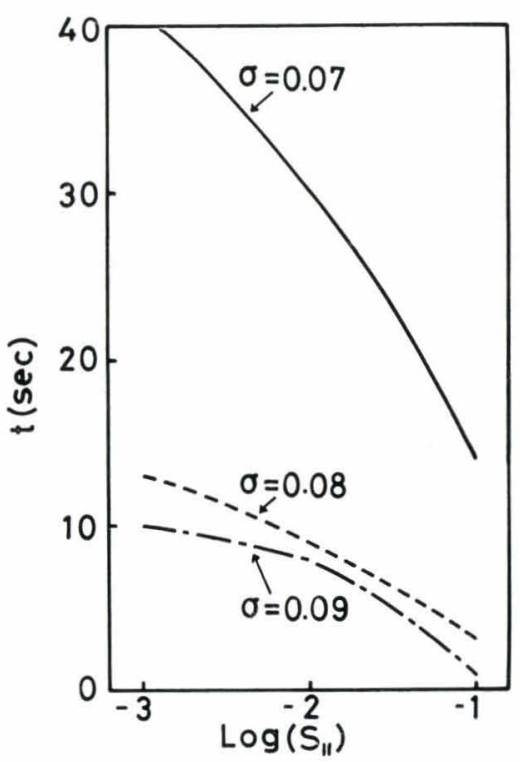

Fig. 6. Relationship between the dendrite arm spacing $\left(S_{\text {II }}\right)$ and the elapsed time $(t)$ at which the instability of system occurs firstly 
stability has been quantitatively investigated by using the perturbation theory on the basis of the mathematical model presented in this paper. Further, the model experiments were conducted to examine the analytical results regarding the formation of the channel-type segregation. Then the followings are clarified.

(1) The channel-type segregation will not be formed if the melt is cooled from the top of mold with its side walls insulated.

(2) When the melt is cooled from the bottom of mold with its side walls insulated, the channel-type segregations may be extensive or slight in accordance with if the disturbance propagates quickly or slowly.

(3) When the melt is cooled from the side walls of the mold, the channel-type segregations can be prevented by providing such alloy elements as to decrease the density of the interdendritic liquid with the increase in the temperature.

The results obtained from the theoretical considerations are qualitatively in good agreement with the empirical results obtained by other investigators.

\section{Nomenclature}

$C:$ concentration $\left(\mathrm{wt}^{\mathrm{o}} \%\right)$

$C_{p}:$ specific heat $\left(\mathrm{cal} / \mathrm{g} \cdot{ }^{\circ} \mathrm{C}\right)$

$D:$ molecular diffusivity $\left(\mathrm{cm}^{2} / \mathrm{sec}\right)$

$G r^{\prime}$ : modified Grashof number (-)

$g$ : acceleration due to gravity $\left(\mathrm{cm} / \mathrm{sec}^{2}\right)$

$\Delta H$ : enthalpy change with freezing (cal $/ \mathrm{g}$ )

$h$ : length of dendrite arm $(\mathrm{cm})$

$K$ : effective distribution coefficient (-)

$k$ : permeability $\left(\mathrm{cm}^{2}\right)$

Le: Lewis number (-)

$L: \quad$ characteristic length $(\mathrm{cm})$

$m$ : slope of the liquidus line $\quad\left({ }^{\circ} \mathrm{C} / \mathrm{wt}^{\mathrm{o}} \%\right)$

$P:$ pressure $\left(\mathrm{g} / \mathrm{cm} \cdot \mathrm{sec}^{2}\right)$

$P e ́$ : Péclet number (-)

Pr: Prandtl number (-)

$R e:$ Reynolds number $=V L \rho / \mu \quad(-)$

Sc: Schmidt number (-)

$S_{v}$ : volumetric specific surface area $\left(\mathrm{cm}^{2} / \mathrm{cm}^{3}\right)$

$S_{\text {II }}$ : secondary dendrite arm spacing $(\mathrm{cm})$

T: temperature $\left({ }^{\circ} \mathrm{C}\right)$

$T^{\prime}$ : small perturbation in temperature $\left({ }^{\circ} \mathrm{C}\right)$

$T_{i}$ : initial temperature $\left({ }^{\circ} \mathrm{C}\right)$

$T_{i}^{\prime}$ : initial small perturbation in temperature $\left({ }^{\circ} \mathrm{C}\right)$

$T_{l}$ : liquidus temperature $\left({ }^{\circ} \mathrm{C}\right)$

$T_{0}$ : melting temperature of a pure material $\left({ }^{\circ} \mathrm{C}\right)$

$T_{o}$ : temperature without perturbation $\left({ }^{\circ} \mathrm{C}\right)$

$T_{s}$ : solidus temperature $\left({ }^{\circ} \mathrm{C}\right)$

$t$ : elapsed time (sec) $t_{p}:$ partial solidification time (sec)

$u$ : velocity in $X$-direction $(\mathrm{cm} / \mathrm{sec})$

$u^{\prime}:$ perturbed velocity in $X$-direction $(\mathrm{cm} / \mathrm{sec})$

$V:$ characteristic velocity $(\mathrm{cm} / \mathrm{sec})$

$v$ : velocity in $Y$-direction $(\mathrm{cm} / \mathrm{sec})$

$v^{\prime}$ : perturbed velocity in $Y$-direction $(\mathrm{cm} / \mathrm{sec})$

$X$ : horizontal co-ordinate $(\mathrm{cm})$

$X_{w}$ : half width of mold $(\mathrm{cm})$

$x$ : dimensionless length (-)

$Y:$ vertical co-ordinate $(\mathrm{cm})$

$Y_{L}:$ length of mold (cm)

$\beta$ : volumetric expansion coefficient of saturated liquid upon freezing $\left(1 /{ }^{\circ} \mathrm{C}\right)$

$\delta(Y)$ : delta function $(-)$

$\varepsilon$ : liquid fraction $(-)$

$\kappa$ : thermal conductivity $\left(\mathrm{cal} / \mathrm{cm} \cdot \mathrm{sec} \cdot{ }^{\circ} \mathrm{C}\right)$

$\lambda$ : thermal diffusivity $\left(\mathrm{cm}^{2} / \mathrm{sec}\right)$

$\mu:$ viscosity $(\mathrm{g} / \mathrm{cm} \cdot \mathrm{sec})$

$\rho:$ density $\left(\mathrm{g} / \mathrm{cm}^{3}\right)$

$\sigma:$ standard deviation (-)

$\phi:$ stream function $\left(\mathrm{cm}^{2} / \mathrm{sec}\right)$

\section{REFERENCES}

1) I. Kodaira: Tetsu-to-Hagané, 18 (1932), 548.

2) I. Hagiwara and T. Takahashi: Tetsu-to-Hagané, 53 (1967), 27.

3) A. Ohno: Tetsu-to-Hagané, 54 (1968), 401.

4) R. J. McDonald and J. D. Hunt: Trans. AIME, 245 (1969), 1993.

5) K. Narita and M. Taniguchi: Tetsu-to-Hagané, 56 (1970), 212.

6) R. Mehrabian, M. Keane and M. C. Flemings: Met. Trans., 1 (1970), 1209.

7) S. M. Copley, A. F. Giamei, S. M. Johnson and M. F. Hornbecker: Met. Trans., 1 (1970), 2193.

8) K. Suzuki and T. Miyamoto: Report of the 19th Committee, Japan Society for the Promotion of Science, Report No. 9478, (1972).

9) K. Suzuki and T. Miyamoto: Tekko no Gyokogensho, ed. by ISIJ, JIM \& JSPS, (1976), 41.

10) K. Suzuki: Report of the 19th Committee, Japan Society for the Promotion of Science, Report No. 9919, (1976).

11) M. C. Flemings: Scand. J. Met., 5 (1976), 1.

12) C. Benedicks, N. Ericsson and G. Ericson: Arch. Eisenhüttenw., 3 (1930), 473.

13) E. Widawaski and F. Sauerwald: Z. Anorg. Allgem. Chem., 192 (1930), 145.

14) L. D. Lucas: Mem. Sci. Rev. Met., 61 (1964), 97.

15) E. S. Filippov and A. M. Samarin: Fiziko-Khimicheskie Osnovy Proizvodstava Stali, Nauka, Moskva, (1968), 3.

16) A. A. Vertman, E. S. Filippov and A. M. Samarin: $I z v$. VUZ. Chern. Met., (1964), No. 7, 19.

17) C. E. Smeltzer: Iron Age, 184 (1959), No. 9, 188. 